\title{
REGULAR EXPRESSION CROSSWORDS
}

\author{
THINH D. NGUYEN
}

\begin{abstract}
We show that fulfilling a rectangular crossword table with a regular expression for each row and each column is very hard.
\end{abstract}

\section{Problem statement}

Definition 1. Regex Crossword problem:

Input: an $M \times N$ grid with $N$ regular expression for the columns and $M$ for the rows

Output: Yes if it is possible to fulfill the table satisfying all the regular expressions, No otherwise

We reduce Vertex Cover problem to ours. Vertex Cover is shown to be hard in [1].

Claim 2. We have that Vertex Cover $\leq_{p}$ Regex Crossword

\section{Reducing Vertex Cover to Regex Crossword}

The problem is NP-hard. We show this by reducing vertex cover:

Given a graph $G=(V, E)$ and a threshold $k$, is there a subset $V^{\prime} \subseteq V$ of cardinality at most $k$, so that each edge in $E$ is incident to at least one node in $V^{\prime}$ ?

We translate this into a regex crossword with $|E|+1$ columns and $|V|$ rows as follows:

All columns, except for the first, correspond to an edge. They get a regex $0^{*} 1(0 \mid 1)^{*}$.

All rows correspond to a vertex. They get a regex that allows to write either

- a 1 in the first column and each column corresponding to an edge incident to that node and zeroes in all other columns, or

- $0^{*}$

Finally, the first column counts the size of the vertex cover. It gets a regex, that allows for at most $k$ ones.

The correspondence between solutions to the regex crossword and vertex covers should be obvious.

Example:

Find a vertex cover of size 2 for the following graph:

Key words and phrases. alphabet, crossword, graph, string.

Perebor. 


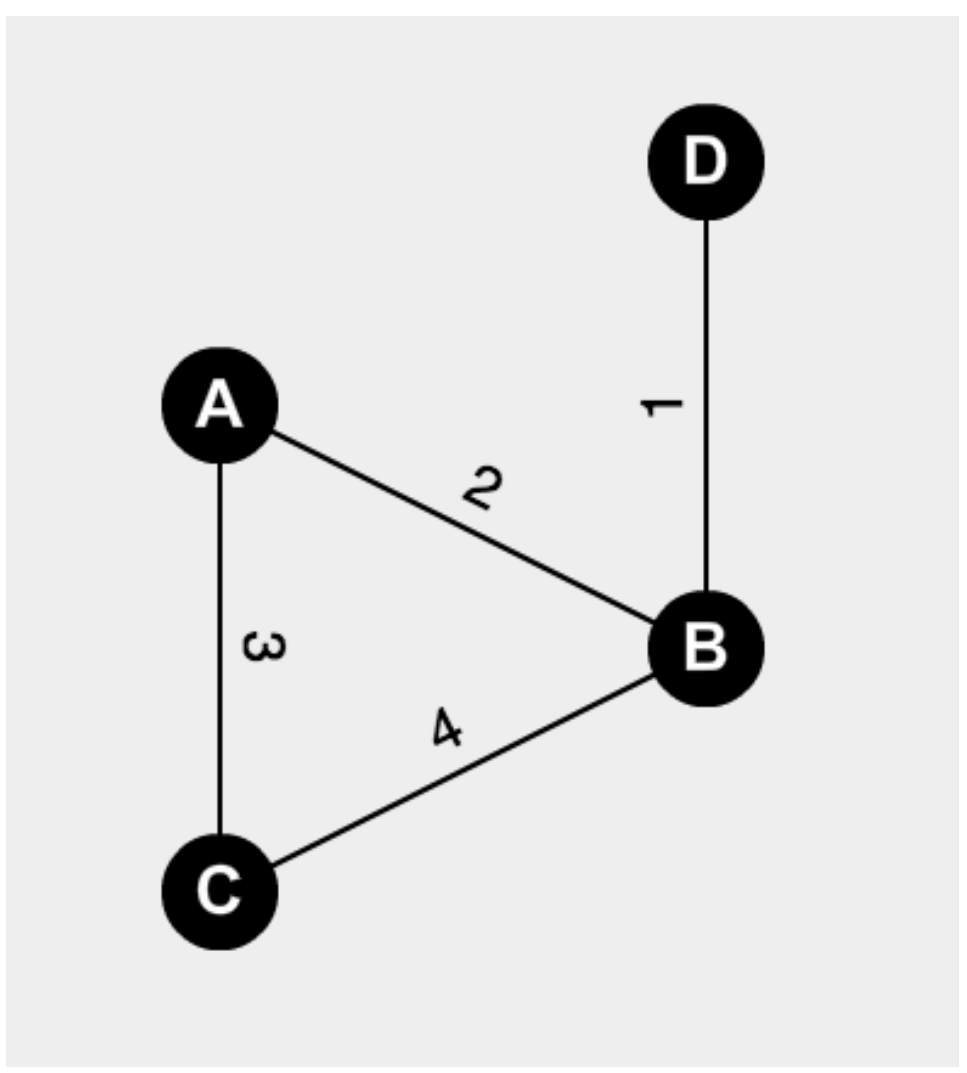

$V_{A}=0^{*} \mid 10110$

$V_{B}=0^{*} \mid 11101$

$V_{C}=0^{*} \mid 10011$

$V_{D}=0^{*} \mid 11000$

Counter $=0^{*}\left|0^{*} 10^{*}\right| 0^{*} 10^{*} 10^{*}$

$E_{1}=0^{*} 1(0 \mid 1)^{*}$

$E_{2}=0^{*} 1(0 \mid 1)^{*}$

$E_{3}=0^{*} 1(0 \mid 1)^{*}$

$E_{4}=0^{*} 1(0 \mid 1)^{*}$

Lastly, we can set up the "crossword" so that $V_{A}$ through $V_{D}$ are the top regexes and Counter and $E_{1}$ through $E_{4}$ are the left side regexes.

Solving this regex crossword give us a vertex cover of size 2 for nodes $V_{A}, V_{B}$ or $V_{C}, V_{B}$.

If we change $k$ to be 1 and Counter to be $0^{*} \mid 0^{*} 10^{*}$ as another example, the regex crossword is impossible to solve because there is no vertex cover of size 1 .

\section{Conclusion}

Garey and Johnson [1] shape their theory based on previous primal works of Cook, Levin and Karp. Johnson [2] moves on with the guide to this theory. As long as we study a mathematical conjecture, we should encourage ourselves to spend enough laboring hours on popular math books like these. Then, reading 
some articles on theory of computing like [3] is a good practice. Only after that, could we think of the ultimate final for all mathematical sciences.

\section{REFERENCES}

1. Michael R. Garey, David S. Johnson, Computers and Intractability: A Guide to the Theory of NP-Completeness

2. David S. Johnson, The NP-Completeness Column: An Ongoing Guide

3. Phan Dinh Dieu, Le Cong Thanh, Le Tuan Hoa, Average Polyno-mial Time Complexity of Some NP-Complete Problems, Theor. Comput. Sci. 46(3): pp.219-237 (1986)

Current address: Department of Mathematics, Moscow State University

Email address: kosmofarmer@yandex.com 